\title{
Retinal Blood Vessel Extraction using Wavelet Decomposition
}

\author{
Diana Tri Susetianingtias ${ }^{1}$ \\ Departement of Computer System \\ Gunadarma University, Depok, Indonesia \\ Sarifuddin Madenda ${ }^{2}$ \\ Doctoral Program in Information Technology \\ Gunadarma University, Depok, Indonesia \\ Fitrianingsih $^{3}$ \\ Departement of Information System Diploma \\ Gunadarma Universiry, Depok, Indonesia
}

\author{
Dea Adlina ${ }^{4}$, Rodiah $^{5}$ \\ Departement of Informatics \\ Gunadarma University \\ Depok, Indonesia
}

\author{
Rini Arianty ${ }^{6}$ \\ Departement of Information System \\ Gunadarma University \\ Depok Indonesia
}

\begin{abstract}
One important part of the eye that is critical for processing visual information before it is sent through the optic nerve to the visual cortex is the retina. The retina of each individual has its own uniqueness that can be used as a characteristic feature in identifying, verifying, and authenticating. The traditional authentication process has various weaknesses such as forgetting the PIN code or losing the ID card used for obtaining system authentication. The results of extracted retinal blood vessels can be used as a feature in the formation of an individual identification system. In the imaging using a fundus camera, the retina's blood vessel has distinguishing shape and number of candidates from one human retina to another. In this research, researchers will develop an algorithm for extracting the retinal fundus image's blood vessels. The feature extraction is done by taking the fundus image feature which is the blood vessel as one of the unique characteristics in forming an individual identification system. The number of blood vessel candidates will then be calculated from the extracted blood vessel result. This research uses wavelet function by looking at the very complex texture of blood vessels using the approximation coefficient. The direction detail coefficient on the wavelet is also used to perform the extraction of retinal blood vessels where the structure of the retinal blood vessels in the fundus image is in all directions. The results of these blood vessel candidates will be used in further research to formulate a biometric system that is formed by unique features in the retinal fundus image which will be used to identify individuals using body traits.
\end{abstract}

Keywords-Blood vessels; extraction; fundus retina; identification; wavelet

\section{INTRODUCTION}

Currently, individual identification is of great importance [1] since many systems require legitimate users in access control [2], especially for systems that store valuable documents and important data. One identification technology [3] that is presently developing is biometric feature-based identification technology. The biometric identification system is a system that does identification and recognition using a biometric characteristic pattern [4] that one's owned. Previous research on biometrics states that biometrics can be used to increase the security, convenience, and accountability of a system [4] during the authentication detection process and prevent fraud. Determining the exact features for the formation of a biometric system [5] becomes very important in producing a biometric system that is able to identify individuals [6] correctly.

Biometric methods can be a security solution [7] in the authentication process. Biometrics is a technique in evaluating and analyzing biological data [8] from various biometric characteristics [9] such as biometrics of fingerprint, palm, face, retina, and iris. The biometric system-based individual identification technique that is developing today is fingerprints. This is because in a fingerprint there are about 40 unique characteristics [10], which enable the identification of about 1.1 trillion different individuals. Apart from fingerprints, one of the body parts that can be used as a biometric system for identification is the retina. The retina is a sensitive eye organ [11] and it functions in the ability to see. Aside from being used to see, the retina can be used as identification [12] as it has unique characteristics. In the retinal tissue of the human eye [13], there are about 256 unique characteristics. The retina has several special characteristics such as the pattern of blood vessels. It differs in each individual even in humans who are identical twins, both on the left and right eyes. Hence, that features [14] on the retina can be used as a sufficiently reliable biometric system. The results of the retinal blood vessel pattern segmentation can be used as a characteristic feature in the formation of the retinal biometric system to identify individuals. The division of 4 quadrants in the fundus image is done by using the Field of View (FOV) approximation of the optical disc [15].

Retinal artery extraction research [16] was carried out using the Kekre's Fast Codebook Generation (KCFG) Algorithm. This research forms a vector that consists of 
candidates for deep blood vessels used as a training set in the initial cluster. The first element of the training set is compared to the first element in the code vector to break the cluster into two. This study [17] uses several iterations to obtain blood vessel candidates. This retinal blood vessels extraction research was carried out using the Isotropic Undecimated Wavelet Transform. The researchers combined the transformation of IUWT, background noise and Gaussian filters to extract retinal blood vessels. The results of this study were then compared with the results of examinations by experts and obtained reliable results in extracting retinal blood vessels with an accuracy of $95 \%$. This research [18] of retinal blood vessel image extraction was carried out by filtering through the convolution process between $\mathrm{f}(\mathrm{x}, \mathrm{y})$ images with the Hessian of Gaussian matrix. The Hessian of Gaussian is obtained by a partial derivative and a second derivative of the Gaussian function at each point of the fundus image that illustrates variations in blood vessel intensity. Researchers used several elements in the hessian matrix to extract blood vessels in the direction of axis $x$, axis $y$ and extraction of the blood vessels in two diagonal directions.

In this study, extraction of blood vessels from retinal fundus images used wavelet decomposition. The results of blood vessel extraction will then be counted as blood vessel candidates. The number of segments is calculated using connected component analysis. The results of this study are expected to produce an extracted image of blood vessels that will be used as one of the features in the formation of the retinal biometric system.

\section{EASE OF USE}

The stages in extracting blood vessels begin with the extraction preprocessing by eliminating other objects in the retinal fundus image such as optical disc. Blood vessels extraction in this study was conducted by researchers using wavelet decomposition by looking at the structure of blood vessels in fundus images with variations of texture in blood vessels.

\section{A. Preprocessing of Blood Vessels Extraction}

Preprocessing is conducted by deleting other objects in the fundus image aside from blood vessels such as optical disc and fundus image background. The initial stage is done by image binarization. After the image is binaryised, then green channel extraction is done wherever the composition of the green channel in the retinal fundus image is at the right saturation [19], the extraction is then used in the next process. Afterwards, Histogram Equalization is performed so that each images has a histogram with a uniform image gray level distribution by mapping each pixel value in the initial histogram to a new pixel value. Histogram equalization is conducted to adjust the pixel values so that produced images has better contrast. The researcher then performs the opening operation $\mathrm{A}^{\circ} \mathrm{B}$ to remove the optical disc with a round shape that seems bright [20] through the erosion process of $\mathrm{A} \Theta \mathrm{B}$ followed by the operator $\oplus$ to dilates based on the structuring element $\mathrm{B}$ as in the formula:

$A^{\circ} B=(A \Theta B) \oplus B$

\section{B. Blood Vessels Extraction using Wavelet Decomposition}

After the edge of the fundus image is removed, the next action is to extract the retinal blood vessels by these following steps:

1) Determine two threshold values $T_{1}$ and $T_{2}$ where $T_{1}>$ $\mathrm{T}_{2}$

2) Every edge pixel with a value greater than $T 1$ is retained as edge pixels.

3) Edge pixels located around step 2 edge pixels with value greater than $\mathrm{T} 1$ threshold are also retained as edge pixels if the value is greater than T2. The goal is to determine the percentage of pixels that are maintained as a segment of a vein.

4) Perform noise elimination (objects outside the blood vessel candidates) so that the extracted image leaves only objects that are blood vessels and removes small objects such as exudates (soft exudates / hard exudates), microaneursym. This process is done by:

a) Determining the minimum object size and minimum hole size.

b) Removing small objects if the area of the object is more than or equal to the minimum object size.

c) Filling small holes if the hole area is less than the minimum hole size.

d) Filtering with Gaussian Filter using formula (2):

$G(x, y)=\frac{1}{2 \pi \sigma^{2}} \exp \left(-\left(x^{2}+y^{2}\right) / 2 \sigma^{2}\right)$

Where $\sigma$ is the standard deviation of the functions distribution in formula (2), with the center of distribution being on the line of $x=0($ mean $=0)$.

e) Determining the coefficient of $\mathrm{cA}$ image matrices and distance coefficients of $\mathrm{cH}, \mathrm{cV}$, and $\mathrm{cD}$ matrices (horizontal, vertical, and diagonal), obtained from wavelet decomposition to maintain blood vessels candidates. The coefficients [cA, cH, cV, cD] will compute the estimated coefficient of the $\mathrm{cA}$ image matrix and the distance coefficients of the $\mathrm{cH}, \mathrm{cV}$, and $\mathrm{cD}$ matrix (horizontal, vertical, and diagonal), obtained from wavelet decomposition of the input image matrix as can be seen in the Fig. 1.

If 2-dimensional discrete wavelet transformation process is conducted to an image with one decomposition level, it will produce four subband as can be seen in Fig. 2, which includes:

1) Approximation coefficient $(\mathrm{CA} \mathrm{j}+1)$ or also called $\mathrm{LL}$ subband

2) Horizontal Detail Coefficient $(\mathrm{CD}(\mathrm{h}) \mathrm{j}+1)$ or also called HL subband

3) Vertical Detail Coefficient $(C D(v) j+1)$ or also called the LH subband

4) Diagonal Detail Coefficient $(\mathrm{CD}(\mathrm{d}) \mathrm{j}+1)$ or also called HH subband 
Two-Dimensional DWT

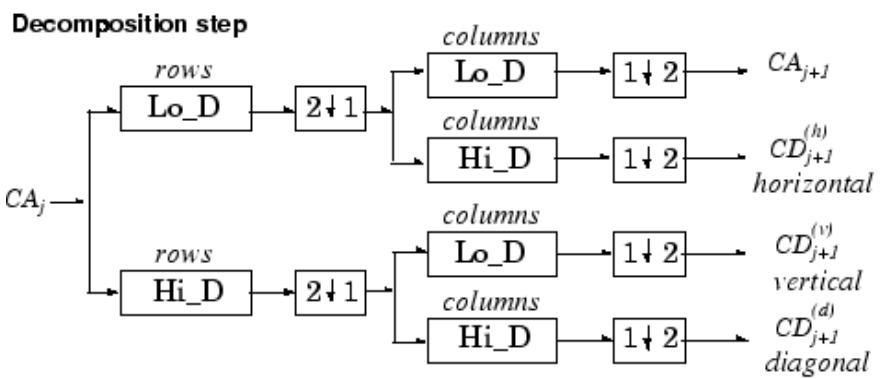

Where $2 \downarrow 1$ Downsample columns: keep the even indexed columns

$1 \downarrow 2$ Downsample rows: keep the even indexed rows

rows
$\mathrm{X}$ Convolve with filter $\mathrm{X}$ the rows of the entry
columns
$\mathrm{X}$ Convolve with filter $\mathrm{X}$ the columns of the entry

Initialization

$C A_{0}=s$ for the decomposition initialization

Fig. 1. DWT Step [20].

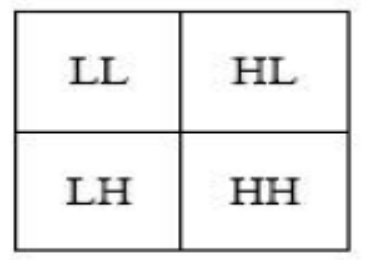

Fig. 2. Wavelet Coefficient Subband.

Decomposition level 1 subband resulting from a decomposition can be decomposed again, because wavelet decomposition level is valued from 1 to $n$. If another decomposition is performed, the LL subband will be decomposed because the LL subband contains most of the image information. If decomposition is done with two decomposition level, the LL subband will produce four new subbands, namely the LL2 sub-band (Approximation Coefficient 2), HL2 (Horizontal Detail Coefficient 2), LH2 (Vertical Detail Coefficient 2), and HH2 (Diagonal Detail Coefficient 2), and so on if another decomposition is to be done.

If an original image $\mathrm{f}$ with $\mathrm{M} \times \mathrm{N}$ pixel is decomposed, it will be decomposed into four subband according to its frequency namely LL, LH, HL, and $\mathrm{HH}$ using wavelet transform. Wavelet transform is derived from a scaling function, whose characteristic is that it can be compiled from a number of copies of itself that have been dilated, translated and scaled. The scaling function $\Psi_{\mathrm{a}, \mathrm{b}}(\mathrm{x})$ is obtained through the translation and dilation of a kernel function $\Psi(\mathrm{x})$ with the formula:

$\Psi_{\mathrm{a}, \mathrm{b}}(\mathrm{x})=\left|\frac{1}{\sqrt{a}}\right|_{\Psi}\left(\frac{x-b}{a}\right)$
Where $\mathrm{a}$ is dilation or scaling parameter $(\mathrm{a} \varepsilon \mathrm{R}), \mathrm{b}$ is translation parameter ( $b \varepsilon R$ ) and $\mathrm{R}$ is a real number. The parameter a shows the width of the Wavelet curve. The parameter $b$ indicates that the localization of the Wavelet curve is centered at the space interval $\mathrm{x}=\mathrm{b}$. By varying parameter a, different frequency resolutions are obtained. Reducing a makes the Wavelet narrower, while making the Wavelet function widens. Scaling function that can form a Wavelet in this study uses a cubic B-Spline Wavelet which has a scaling function with coefficient $c_{0}=1 / 16, c_{1}=4 / 16, c_{2}$ $=6 / 16, c_{3}=4 / 16$, and $c_{4}=1 / 16$.

\section{Blood Vessel Candidate Calculation}

In order to obtain one of the unique features for forming a biometric system is to calculate the number of candidate blood vessels (vessel segment). Vessel segment is the number of segmented blood vessel images taken from segmentation or in segment candidates. The number of segments is determined using connected component analysis, i.e. if neighboring pixels have the same intensity, then the neighboring pixel will be labeled as a pixel in the region (the same object) of that pixel. Marking is done by examining pixels to identify the area of connected pixels as can be seen in Fig. 3.

1) Determining the characteristic of blood vessels was conducted as follows:

- For a vein in the form of a straight line, assume that the vein is a line that has Mid Point coordinates. The midpoint of the line in the vein is the point located in the middle of the two end points. The midpoint is the average of the two end points which are the average of two $x$ coordinates and two y coordinates determined by the formula:

$\left[\left(\frac{x_{1}+x_{2}}{2}\right),\left(\frac{y_{1}+y_{2}}{2}\right)\right]$

Blood vessels consist of 2 Edge Points. For example Edge Point $A=\left(x_{1}, y_{1}\right)$ and Edge Point $B=x_{2}, y_{2}$ as can be seen in Fig. 4.

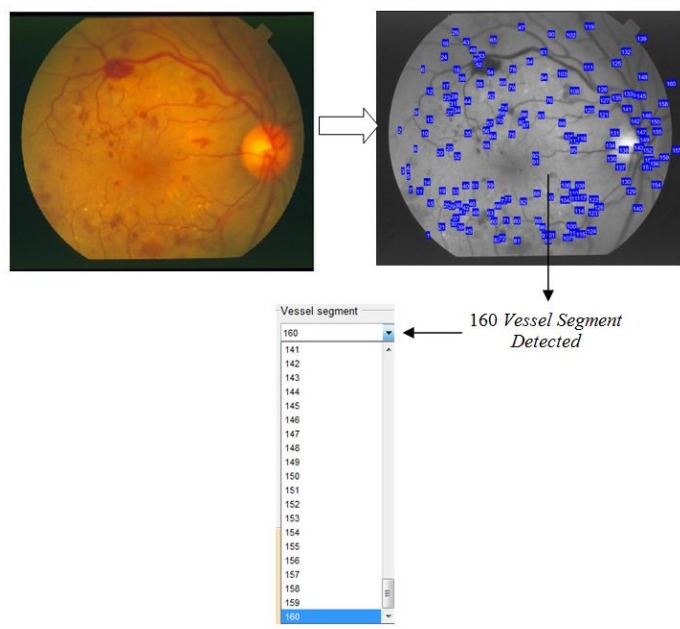

Fig. 3. Vessel Segment Component Labelling. 


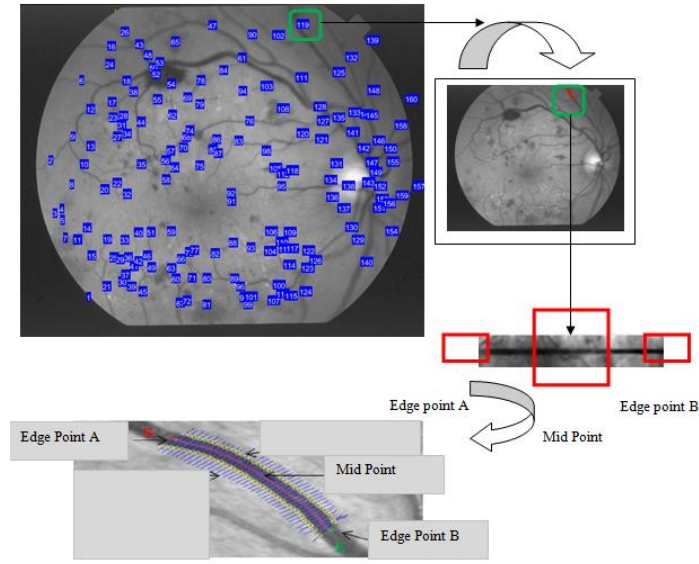

Fig. 4. Blood Vessel Coordinate Determination.

\section{RESUlT AND DISCUSSION}

The use of the Wavelet function in this study was carried out by the researchers because the texture of candidate blood vessels images has many vessels with uniform texture. Image with texture uniformity has very little changes in gray value, thus having a large energy. Conversely, heterogeneous images have a lot of gray value changes so that the energy value is small. The energy value itself is taken from 4 values which are the approximation coefficient (ca), the horizontal direction detail coefficient (ch), the vertical direction detail coefficient (cv), and the diagonal direction detail coefficient (cd) whose value depends on the value of the GS. The results of the process for obtaining blood vessel candidates can be seen in Fig. 5.

In the table there is the original image, the resulting image of the candidate blood vessels and the blood vessel extraction image obtained using Wavelet decomposition to acquire the entire blood vessels (arteries or veins).

A fundus image was tested to extract blood vessels of the retinal fundus as can be seen in Table I. In the table there is the original image, the resulting image of the candidate blood vessels and the blood vessel extraction image obtained using Wavelet decomposition to acquire the entire blood vessels (arteries or veins).

(a) Original Image
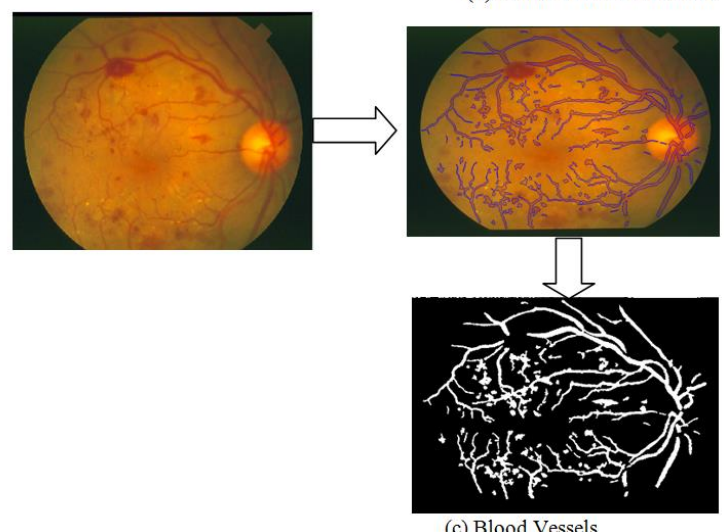

(c) Blood Vessels

(b) Blood Vessels Candidate

Fig. 5. Retina Blood Vessel Extraction Process.
TABLE I. EXAMPLE OF BLOOD VESSEL EXTRACTION RESULTS

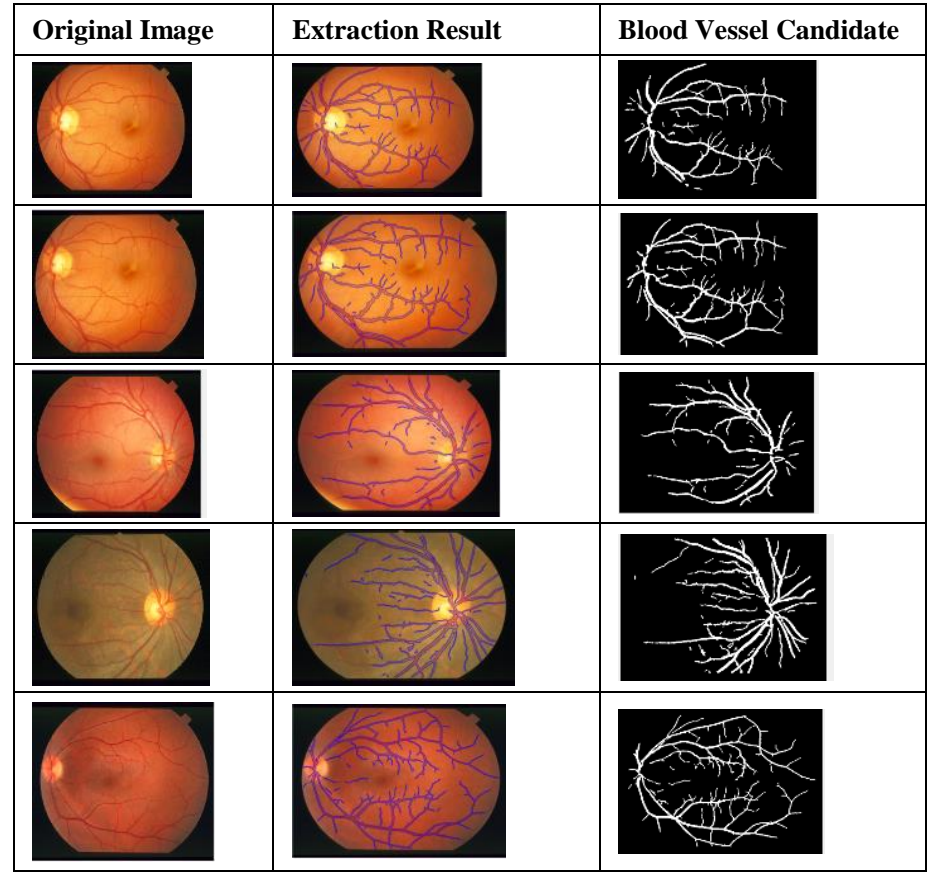

As can be seen in Table II, fundus images will be labeled and the number of segmented blood vessel candidates will be counted.

As can be seen in Table II, five fundus images are given as example. Each of which will be labeled and the number of segmented blood vessel candidates will be counted. It can be seen that the implemented algorithm successfully determines the number of extracted blood vessel candidates.

TABLE II. EXAMPLE OF IMAGE LABELING RESULTS AND NUMBER OF BLOOD VESSEL CANDIDATES

\begin{tabular}{|c|c|c|}
\hline $\begin{array}{l}\text { Original } \\
\text { Image }\end{array}$ & $\begin{array}{l}\text { Labeled } \\
\text { Image }\end{array}$ & $\begin{array}{l}\text { Blood Vessel } \\
\text { Candidate }\end{array}$ \\
\hline & & 171 \\
\hline 18 & 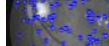 & 133 \\
\hline & & 130 \\
\hline 8 & & 166 \\
\hline & & 161 \\
\hline
\end{tabular}




\section{CONCLUSION}

The extraction algorithm done by the researchers succeeded in removing the edge of the retina that is not included as the vessels (Field of View) and obtaining candidates for retinal blood vessels. This number of candidates' results will be used as one of the features in the formation of the retinal biometric system. Further development can be done by determining other unique features where these features will be used as attributes in the retinal biometric identification system so that the accuracy in an individual recognition is improved.

\section{ACKNOWLEDGMENT}

Thank you to DP2M Ristekdikti for research funding in the applied scheme. A sincere gratitude to Gunadarma University especially to the Gunadarma University Research Bureau for the opportunity to conduct research specifically in the field of Biometrics.

\section{REFERENCES}

[1] Hazem M. El-Bakry, Mohy A. Abou-Elsoud, and Mohamed S. Kamel, 2000, A Biometric System For Personal Identification Using Modular Neural Nets, Mansoura Engineering Journal - Mansoura University EGPYT.

[2] Ashbourn, J, 2004, Practical Biometrics: from Aspiration to Implementation, Springer professional computing, London: Springer.

[3] Kalyani CH, 2017, Various Biometric Authentiocation Techniques A Review, Journal of Biometrics \& Biostatistics, Vol 8 : 371. ISSN. 21556180.

[4] Unar JA, Chaw Seng W, Abbasi A, 2014, A review of biometric technology along with trends and prospects. Pattern recognition 47: 2673-2688.11.

[5] Prins,C, 1998, Biometric Technology Law, Making Our Body Identify for Us: Legal Implications ofBiometric Technologies, Computer Law \& Security Report Vol.14 no.3.

[6] Soutar, C, 2002, Biometric System Security, in: Secure No. 5, p 46-49.
[7] Wacks, R, 1989, Personal Information: Privacy and the Law, Oxford: Clarendon Press.

[8] Tuyls,P and Goseling, J.(2004), Capacity and Examples of Template Protecting Biometric Authentication Systems. D. Maltoni and AK Jain (Eds): BioAW, LNCS3087, pp158-170.

[9] A. Ahmed and I. Traore. Anomaly intrusion detection based on biometrics, 2005, In 6th IEEE Information Assurance Workshop.

[10] Edmund Spinella, 2003 Biometric Scanning Technologies: Finger, Facial, and Retinal Scanning. SANS Institute.

[11] Brad Bowling. Kanskis's Clinical Ophthalmology A Systematic Approach, Eighth Edition, ISBN: 978-0-7020-5572-0, Elsevier, Sydney, New South Wales, Australia. 2016.

[12] Julian Ashbourn. Biometrics: Advanced Idendity Verification - The Com- plete Guide. Springer-Verlag, London, Great Britain, 2002.

[13] Mudholkar, Shende, Sarode, 2012, Biometrics Authentication Technique For Intrusion Detection Systems Using Fingerprint Recognition, International Journal of Computer Science, Engineering and Information Technology. Vol 2, No.1.

[14] Zhenchang Wang, Junfang Xian, Fengyuan Man, Zhengyu Zhang, 2018, Diagnostic Imaging of Ophthalmology: A Practical Atlas 1st ed, Springer.

[15] Fahreddin Sadikoglu, Selin Uzelaltinbulat, 2016, Biometric Retina Identification Based on Neural Network, Procedia Computer Science, Volume 102, Pages 26-43.

[16] Borah, Sarma, Talukdar, 2015, Retina Recognition System using Adaptive Neuro Fuzzy Inference System, IEEE International Conference on Computer, Communicatioan and Control.

[17] Sasidharan, Retinal Based Personal Identification System Using Skeletonizatioan and Similarity, 2014, International Journal of Computer Trends and Technology (IJCTT)-vol.17, number 3.

[18] Joddat Fatima, Adeel M. Syed, M. Usman Akram. A Secure Personal Identification System Based on Human Retina. Bahria University, Pakistan. 2013.

[19] https://www.isi.uu.nl/Research/Databases/DRIVE/. Accesed Date: Mei 5, 2018.

[20] Diana Tri Susetianingtias, Suryadi Hs, Sarifuddin Madenda, Rodiah, Fitrianingsih, 2017, Blood Vessel Extraction And Bifurcations Detection Using Hessian Matrix Of Gaussian And Euclidian Distance, Journal Of Theoretical And Applied Information Technology. 